Published in final edited form as:

Target Oncol. 2016 April ; 11(2): 229-234. doi:10.1007/s11523-015-0389-2.

\title{
Effect of renal impairment on the pharmacokinetics and safety of axitinib
}

\author{
Ying Chen ${ }^{1}$, Brian I. Rini ${ }^{2}$, Robert J. Motzer ${ }^{3}$, Janice P. Dutcher ${ }^{4}$, Olivier Rixe ${ }^{5}$, George \\ Wilding $^{6}$, Walter M. Stadler ${ }^{7}$, Jamal Tarazi ${ }^{1}$, May Garrett ${ }^{1}$, and Yazdi K. Pithavala ${ }^{1}$ \\ ${ }^{1}$ Pfizer Oncology, San Diego, CA \\ ${ }^{2}$ Cleveland Clinic Taussig Cancer Institute, Cleveland, $\mathrm{OH}$ \\ ${ }^{3}$ Memorial Sloan Kettering Cancer Center, New York, NY \\ ${ }^{4}$ New York Medical College, Valhalla, NY \\ ${ }^{5}$ University of New Mexico Cancer Center, Albuquerque, NM \\ ${ }^{6}$ University of Wisconsin Carbone Comprehensive Cancer Center, Madison, WI \\ ${ }^{7}$ Department of Medicine, University of Chicago, Chicago, IL
}

\begin{abstract}
Background-Axitinib, an inhibitor of vascular endothelial growth factor (VEGF) receptors, is approved as second-line treatment for advanced renal cell carcinoma (RCC). Agents targeting the VEGF pathway may induce renal toxicities, which may be influenced by pre-existing renal dysfunction.

Objective-To characterize axitinib pharmacokinetics and safety in patients with renal impairment.
\end{abstract}

Patients and Methods-Effect of renal function (baseline creatinine clearance [CrCL]) on axitinib clearance was evaluated in a population pharmacokinetic model in 207 patients with advanced solid tumors who received a standard axitinib starting dose, and 383 healthy volunteers. Axitinib safety according to baseline CrCL was assessed in previously treated patients with RCC $(n=350)$ who received axitinib in the phase 3 AXIS study.

Results-Median axitinib clearance was $14.0,10.7,12.3,7.81$, and $12.6 \mathrm{~L} / \mathrm{h}$, respectively, in individuals with normal renal function ( $290 \mathrm{ml} / \mathrm{min} ; n=381$ ), mild renal impairment $(60-89 \mathrm{ml} /$ min; $n=139)$, moderate renal impairment (30-59 ml/min; $n=64)$, severe renal impairment (15-29 $\mathrm{ml} / \mathrm{min} ; n=5)$, and end-stage renal disease $(<15 \mathrm{ml} / \mathrm{min} ; n=1)$. The population pharmacokinetic model adequately predicted axitinib clearance in individuals with severe renal impairment or endstage renal disease. Grade $\geq 3$ adverse events (AEs) were reported in $63 \%$ of patients with normal

Corresponding author: Yazdi K. Pithavala, Clinical Pharmacology, Pfizer Inc, 10555 Science Center Drive, San Diego, CA 92121, USA; phone: 858-622-8896; yazdi.pithavala@pfizer.com.

Compliance with Ethical Standards

Olivier Rixe and George Wilding have no conflicts of interest to disclose. 
renal function or mild impairment, $77 \%$ with moderate impairment, and $50 \%$ with severe impairment; study discontinuations due to AEs were $10 \%, 11 \%$, and $0 \%$, respectively.

Conclusions-Axitinib pharmacokinetics and safety were similar regardless of baseline renal function; no starting-dose adjustment is needed for patients with pre-existing mild-to-severe renal impairment.

\section{Introduction}

Axitinib, a potent, selective inhibitor of vascular endothelial growth factor (VEGF) receptors [1], is approved for second-line treatment of advanced renal cell carcinoma (RCC) in many countries. It is well known that treatment with antiangiogenic drugs, including those that target the VEGF pathway, may result in renal toxicities, such as proteinuria. In phase 3 trials of agents targeting the VEGF pathway in patients with metastatic RCC (mRCC), proteinuria was more frequently observed with the monoclonal antibody to VEGF (bevacizumab) than with tyrosine kinase inhibitors of VEGF receptors [2]. Although not reported in large clinical studies, cases of thrombotic microangiopathy, interstitial nephritis, and glomerulonephritis have been described in patients with cancer treated with VEGF pathwaytargeted therapies and are usually reversible following treatment discontinuation [3-5]. These findings underscore the importance of evaluating patients for renal disease prior to initiating antiangiogenic therapy and monitoring renal function during treatment [4].

Knowledge of the pharmacokinetics and disposition of VEGF receptor tyrosine kinase inhibitors, such as axitinib, and anticipated toxicities can aid in development of dosing strategies to balance efficacy and safety [6]. The pharmacokinetics of axitinib have been well characterized in both healthy volunteers and patients with cancer [6, 7]. Axitinib is metabolized predominantly in the liver by cytochrome P450 (CYP) 3A4/5, and marginally by CYP1A2, CYP2C19 and uridine diphosphate glucuronosyltransferase (UGT) 1A1 [7]. The two major circulating metabolites in human plasma are the sulfoxide metabolite and the glucuronide conjugate, which are products of the enzymatic actions of CYP3A4/5 and UGT1A1, respectively; these metabolites are considered pharmacologically inactive, since they exhibit approximately 400- and 8,000-fold less in vitro potency, respectively, against VEGF receptor 2 compared with axitinib [7]. Elimination of axitinib primarily by hepatobiliary excretion prompted a clinical study to evaluate changes in axitinib pharmacokinetics with hepatic impairment. Whereas axitinib plasma exposure following a single 5-mg dose was similar in individuals with mild hepatic impairment and those with normal hepatic function, there was a two-fold increase in plasma exposure in individuals with moderate hepatic impairment [8]. Accordingly, axitinib dosing guidelines recommend the starting dose be reduced by approximately half in patients with moderate hepatic impairment [9].

In the human mass balance study, no unchanged axitinib was detected in urine [10]. Furthermore, in the first-in-human study of axitinib, $<1 \%$ of the administered dose appeared as unchanged drug in urine during 12-h urinary collection in patients with advanced solid tumors [11]. Together, these data indicate there is minimal involvement of the renal pathway in the overall elimination of axitinib, and a formal study of the effects of renal impairment 
on axitinib pharmacokinetics and safety was not performed. Preclinical studies of various drugs that are eliminated via non-renal pathways have suggested that severely diminished renal function may still result in reduced CYP enzyme and drug transporter activity in the liver and gut wall [12]. In addition, since patients with advanced RCC often present with comorbidities, including impaired renal function secondary to prior nephrectomy, pharmacokinetic and safety investigations in individuals with renal impairment are warranted.

A population pharmacokinetic approach was used to characterize the effect of renal impairment on axitinib disposition. In an analysis using data from 207 patients with advanced solid tumors, including 181 with mRCC, and 383 healthy volunteers, renal function (creatinine clearance $[\mathrm{CrCL}]$ as a continuous variable) was found not to be a significant predictor of axitinib pharmacokinetics in the overall population [13]. To characterize the effect of renal impairment on axitinib pharmacokinetics, we analyzed data from this population pharmacokinetic model according to baseline renal function. In addition, we assessed axitinib safety in patients with mRCC treated with axitinib in the phase 3 AXIS study [14] and axitinib dose titration in patients with mRCC treated with axitinib in phase 2 studies [15-17] according to renal function.

\section{Patients and methods}

\subsection{Pharmacokinetic assessment according to renal function}

The population pharmacokinetic-pharmacodynamic model has been previously described in detail [13]. Briefly, axitinib was rapidly absorbed (absorption rate $=0.482 \mathrm{~h}^{-1}$ ), systemic clearance (CL; interindividual variability [IIV] expressed as percentage coefficient of variation) was estimated to be $14.6 \mathrm{~L} / \mathrm{h}(59.9 \%)$, and central volume of distribution (IIV) was estimated to be $47.3 \mathrm{~L}$ (39.7\%). Here, individuals that were included in the population pharmacokinetic analysis were grouped according to renal function (baseline CrCL, estimated using the Cockcroft-Gault formula and using thresholds from the US Food and Drug Administration [FDA] guidance for evaluating pharmacokinetics in patients with impaired renal function [18]); axitinib clearance (CL) was then summarized for each group. Adequate prediction of axitinib CL in the different renal function categories in our model was assessed by degree of model shrinkage in the random effect, which provides a measure of the extent to which the individual estimates of CL differ from the population-predicted CL value; the a priori cut-off of $\_20 \%$ shrinkage was considered sufficient for predictions of individual estimates of CL $[19,20]$. Other diagnostics, including distribution of random effects on CL and distribution of the observed data compared with the population- and individual-predicted values from the model were also explored.

\subsection{Safety assessment according to renal function}

Data from the AXIS trial (NCT00678392) [14], a randomized, open-label, phase 3 study of axitinib versus sorafenib in patients with $\mathrm{mRCC}$ following failure of one prior systemic firstline treatment, were used to evaluate the safety of axitinib in patients with renal impairment. Adverse events (AEs) were assessed throughout the study, graded according to the Common Terminology Criteria for Adverse Events version 3.0, and evaluated for relationship to the 
study treatment by the investigator [14]. Patients were grouped into renal function categories according to baseline $\mathrm{CrCL}$ and using thresholds defined in the European Medicines Agency guidance on evaluation of the pharmacokinetics of medicinal products in patients with impaired renal function [21]. Treatment-related AEs, grade $\geq 3$ AEs, treatment discontinuations due to AEs, and changes in serum creatinine as well as CrCL from baseline were compared in axitinib-treated patients with moderate and severe renal impairment versus axitinib-treated patients with mild renal impairment or normal renal function. Patients with mild renal impairment were pooled with those with normal renal function in order to adequately discriminate safety findings from the more clinically significant moderate and severe renal impairment groups.

Data from three phase 2 trials of axitinib in patients with cytokine-refractory (NCT00076011; NCT00569946) [15, 16] and sorafenib-refractory (NCT00282048) [17] $\mathrm{mRCC}$ were used to evaluate axitinib dose titration in patients with renal impairment. In all studies, patients were administered axitinib at a starting dose of $5 \mathrm{mg}$ twice daily. Here, patients were grouped into renal function categories according to baseline CrCL using FDA thresholds [18].

\section{Results}

\subsection{Effect of renal impairment on axitinib pharmacokinetics}

Median axitinib CL values were similar across different renal-function groups per baseline CrCL (Table 1). Due to the small size of the population with severe renal impairment or endstage renal disease at baseline ( $n=6)$, we assessed if axitinib CL was adequately predicted in our model by evaluating degree of shrinkage and distribution of random effect on CL (Fig. 1). Model shrinkage for the random effect on CL was low in each renal-function group: $10.6 \%$ in individuals with normal renal function $(n=381), 1.4 \%$ in those with mild renal impairment ( $n=139), 4.2 \%$ in those with moderate renal impairment ( $n=64)$, and $10.8 \%$ in those with severe renal impairment or end-stage renal disease $(n=6)$. The random effects distributions showed no systematic association with renal impairment; in particular, random effects for the severe renal-impairment group exhibited normal distribution and were centered at a mean of zero (Fig. 1).

Diagnostic plots showed agreement between the observed axitinib plasma concentrations and the population and individual predictions, and no systemic bias in the residuals (data not shown). Population pharmacokinetic analysis using lowest CrCL on study, instead of baseline CrCL, yielded similar results (data not shown).

\subsection{Effect of renal impairment on axitinib safety in patients with mRCC}

3.2.1 Phase 3 AXIS trial-Based on baseline CrCL, 73 (21\%) of 350 evaluable axitinibtreated patients in the phase 3 AXIS study had moderate renal impairment $(30-50 \mathrm{ml} / \mathrm{min})$, and $4(1 \%)$ had severe renal impairment $(<30 \mathrm{ml} / \mathrm{min})$. All other axitinib-treated patients had mild renal impairment or normal renal function $(n=273)$.

Treatment-related AEs were reported in 243 (89\%) patients with mild renal impairment or normal renal function, 71 (97\%) with moderate renal impairment, and $3(75 \%)$ with severe 
renal impairment. Grade $\geq 3$ AEs were reported in $173(63 \%), 56(77 \%)$, and $2(50 \%)$ patients, respectively, in these renal function groups. In all, $26(10 \%)$ patients with mild renal impairment or normal renal function, $8(11 \%)$ with moderate renal impairment, and no patients with severe renal impairment discontinued axitinib treatment due to AEs. Change in serum creatinine and CrCL from baseline was similar in patients with severe renal impairment and those in other renal-function groups (Table 2).

3.2.2 Phase 2 trials-Of 174 evaluable patients with refractory mRCC who were treated with axitinib in phase 2 studies, 32 had normal renal function, 79 had mild renal impairment, 58 had moderate renal impairment, and 5 had severe renal impairment based on baseline $\mathrm{CrCL}$; the axitinib dose was titrated above 5-mg twice daily in $47 \%, 32 \%, 28 \%$, and $40 \%$ of patients, respectively, in these renal-function groups. Findings were similar when lowest on-study CrCL was used to classify renal function (data not shown).

\section{Discussion}

Although a formal, prospective study of axitinib in individuals with renal impairment has not been conducted, nonclinical and clinical data indicated that the renal system plays a negligible role in axitinib elimination [10]. The analyses presented here provide further evidence that axitinib pharmacokinetics and tolerability are unaffected by renal dysfunction and substantiate recommendations that starting dosing modifications are not warranted in patients with pre-existing mild to severe renal impairment [9].

In the prior report of this population pharmacokinetic model [13], renal function (CrCL) was not identified as a significant covariate on axitinib CL during formal covariate search; however, axitinib pharmacokinetics in patients of varying renal function were not assessed. Here, we extended the previous analysis [13] by classifying individuals treated with axitinib according to baseline renal function and analyzing drug CL in each group. In doing so, we confirmed that axitinib pharmacokinetics were not altered by renal impairment. The similarity of axitinib CL in individuals with severe renal impairment and those with normal renal function was not surprising based on the observation that axitinib is not eliminated through the kidneys in humans [10]. Due to the small number of individuals with severe renal impairment included in the population pharmacokinetic analysis, we further characterized if axitinib CL was adequately predicted by the model in the small population. Consistent with low model shrinkage (7.6\%) for the random effect on CL in the overall population [13], we found shrinkage was low in each renal-function group, indicating that the estimated random effects are considered to have good diagnostic value. In addition, the random effects distributions show no systematic association with renal impairment; specifically, the random effects for the severely impaired group appeared normally distributed and centered at a mean of zero. Based on these diagnostics, we concluded that the population pharmacokinetic model adequately predicted axitinib CL in individuals with severe renal impairment.

In our analysis of renal function and safety using data from the phase 3 AXIS trial [14], which comprises the largest population of axitinib-treated patients in a prospective clinical study, the occurrence of treatment-related AEs was numerically similar in different renal- 
function groups. Patients with severe renal impairment prior to axitinib treatment showed a similar change in serum creatinine and CrCL from baseline to those in other renal-function groups, and laboratory values suggest that worsening of renal disease in these individuals was minimal. Notably, equivalent proportions of patients in different renal-function groups who were treated in phase 2 studies were administered axitinib doses above $5 \mathrm{mg}$ twice daily, which required no grade $>2$ treatment-related AEs for at least 2 weeks [15-17]. This indicates that patients with renal impairment tolerated the drug similarly to those with normal renal function. Together these data suggest that axitinib treatment in patients with compromised renal function is not associated with new safety concerns.

It is recognized that treatment with VEGF pathway-targeted agents may result in renal toxicities, for example proteinuria [2], and cases of thrombotic microangiopathy, interstitial nephritis, and glomerulonephritis have been described in patients with cancer treated with these therapies [3-5]. The effect of renal impairment on the pharmacokinetics of the VEGF receptor inhibitors sunitinib and sorafenib have been evaluated in phase 1 studies. Following a single 50-mg dose of sunitinib [22] or a single 400-mg dose of sorafenib [23],

pharmacokinetics appeared to be similar in individuals with normal renal function and those with severe renal impairment, suggesting dose modifications of these agents are not needed in patients with renal impairment. In addition, prior retrospective analyses have examined the effects of other VEGF pathway-targeted agents that are approved for RCC in patients with diminished renal function. An analysis of 51 patients with mRCC treated with sunitinib, bevacizumab, everolimus, or temsirolimus at a single institution found that efficacy and safety outcomes as well as dose adjustments did not differ substantially in patients with renal impairment (CrCL $\$ 60 \mathrm{ml} / \mathrm{min}$ ) versus those with normal renal function (CrCL $>60 \mathrm{ml} / \mathrm{min}$ ) [24]. Likewise, a retrospective study showed that sunitinib treatment in Korean patients with mRCC and renal insufficiency yielded safety and efficacy outcomes similar to those observed in phase 3 clinical studies [25]. Limited data are available for use of VEGF pathway-targeted agents in patients with $\mathrm{mRCC}$ receiving dialysis, although three small, retrospective analyses suggest that these drugs are tolerated in this patient population [26-28]. Together, these analyses suggest that renal impairment in patients with mRCC does not preclude use of VEGF pathway inhibitors. Nevertheless, due to the observed occurrence of renal toxicities following treatment with these drugs [3-5], it is recommended that patients treated with axitinib, sunitinib, or pazopanib be monitored for urine protein $[9,29$, 30].

The results reported here are limited by the post hoc nature of the analyses and the small number of patients with severe renal impairment or end-stage renal disease. It would be beneficial to evaluate axitinib safety and pharmacokinetics in a larger cohort of patients, including those undergoing renal dialysis for end-stage renal disease. Additionally, categorized renal impairment was not formally tested as a covariate in the population pharmacokinetic model; however, based on the data reported here, it would not be expected to significantly affect axitinib pharmacokinetics. Notwithstanding these limitations, our data indicate that mild to severe renal impairment does not impact axitinib pharmacokinetics or safety; thus, adjustments of the starting dose of axitinib are not needed in this patient population. 


\section{Acknowledgments}

This study was sponsored by Pfizer. Medical writing support was provided by Joanna Bloom, PhD, of Engage Scientific Solutions, and was funded by Pfizer. Ying Chen, Jamal Tarazi, May Garrett, and Yazdi K. Pithavala are employees of and own stock in Pfizer. Brian I. Rini has received grants, consulting fees or honorarium, support for travel to meetings for the study, manuscript preparation, or other purposes, and provision of writing assistance, medicines, equipment, or administrative support from Pfizer. Robert J. Motzer has received grants and consulting fees or honorarium from Pfizer. Janice P. Dutcher has received grants from Pfizer, fees for speakers bureaus and travel support for lectures from Pfizer, Novartis, and Prometheus, and fees for participation on data and safety monitoring boards from Bristol-Myers Squibb, Merck, PrECOG, and Tracon. Walter M. Stadler's institution received funding from Pfizer to conduct the clinical trial.

\section{References}

1. Hu-Lowe DD, Zou HY, Grazzini ML, Hallin ME, Wickman GR, Amundson K, Chen JH, Rewolinski DA, Yamazaki S, Wu EY, McTigue MA, Murray BW, Kania RS, O'Connor P, Shalinsky DR, Bender SL. Nonclinical antiangiogenesis and antitumor activities of axitinib (AG-013736), an oral, potent, and selective inhibitor of vascular endothelial growth factor receptor tyrosine kinases 1 , 2, 3. Clin Cancer Res. 2008; 14:7272-7283. DOI: 10.1158/1078-0432.CCR-08-0652 [PubMed: 19010843]

2. Cohen RB, Oudard S. Antiangiogenic therapy for advanced renal cell carcinoma: management of treatment-related toxicities. Invest New Drugs. 2012; 30:2066-2079. DOI: 10.1007/ s10637-012-9796-8 [PubMed: 22327313]

3. Kelly RJ, Billemont B, Rixe O. Renal toxicity of targeted therapies. Target Oncol. 2009; 4:121-133. DOI: 10.1007/s11523-009-0109-x [PubMed: 19421832]

4. Izzedine H, Massard C, Spano JP, Goldwasser F, Khayat D, Soria JC. VEGF signalling inhibitioninduced proteinuria: Mechanisms, significance and management. Eur J Cancer. 2010; 46:439-448. DOI: 10.1016/j.ejca.2009.11.001 [PubMed: 20006922]

5. Usui J, Glezerman IG, Salvatore SP, Chandran CB, Flombaum CD, Seshan SV. Clinicopathological spectrum of kidney diseases in cancer patients treated with vascular endothelial growth factor inhibitors: a report of 5 cases and review of literature. Hum Pathol. 2014; 45:1918-1927. DOI: 10.1016/j.humpath.2014.05.015 [PubMed: 25087655]

6. Patson B, R BC, Olszanski AJ. Pharmacokinetic evaluation of axitinib. Expert Opin Drug Metab Toxicol. 2012; 8:259-270. DOI: 10.1517/17425255.2012.652947 [PubMed: 22248343]

7. Chen Y, Tortorici MA, Garrett M, Hee B, Klamerus KJ, Pithavala YK. Clinical pharmacology of axitinib. Clin Pharmacokinet. 2013; 52:713-725. DOI: 10.1007/s40262-013-0068-3 [PubMed: 23677771]

8. Tortorici MA, Toh M, Rahavendran SV, Labadie RR, Alvey CW, Marbury T, Fuentes E, Green M, Ni G, Hee B, Pithavala YK. Influence of mild and moderate hepatic impairment on axitinib pharmacokinetics. Invest New Drugs. 2011; 29:1370-1380. DOI: 10.1007/s10637-010-9477-4 [PubMed: 20596748]

9. Pfizer Laboratories. Pfizer Inc.; 2012. Inlyta ${ }^{\circledR}$ (axitinib) prescribing information. http:// labeling.pfizer.com/ShowLabeling.aspx?id=759 [Accessed 10 April]

10. Smith BJ, Pithavala Y, Bu HZ, Kang P, Hee B, Deese AJ, Pool WF, Klamerus KJ, Wu EY, Dalvie DK. Pharmacokinetics, metabolism, and excretion of [14C]axitinib, a vascular endothelial growth factor receptor tyrosine kinase inhibitor, in humans. Drug Metab Dispos. 2014; 42:918-931. DOI: 10.1124/dmd.113.056531 [PubMed: 24608633]

11. Rugo HS, Herbst RS, Liu G, Park JW, Kies MS, Steinfeldt HM, Pithavala YK, Reich SD, Freddo JL, Wilding G. Phase I trial of the oral antiangiogenesis agent AG-013736 in patients with advanced solid tumors: pharmacokinetic and clinical results. J Clin Oncol. 2005; 23:5474-5483. DOI: 10.1200/JCO.2005.04.192 [PubMed: 16027439]

12. Nolin TD, Naud J, Leblond FA, Pichette V. Emerging evidence of the impact of kidney disease on drug metabolism and transport. Clin Pharmacol Ther. 2008; 83:898-903. DOI: 10.1038/clpt. 2008.59 [PubMed: 18388866]

13. Rini BI, Garrett M, Poland B, Dutcher JP, Rixe O, Wilding G, Stadler WM, Pithavala YK, Kim S, Tarazi J, Motzer RJ. Axitinib in metastatic renal cell carcinoma: results of a pharmacokinetic and 
pharmacodynamic analysis. J Clin Pharmacol. 2013; 53:491-504. DOI: 10.1002/jcph.73 [PubMed: 23553560]

14. Rini BI, Escudier B, Tomczak P, Kaprin A, Szczylik C, Hutson TE, Michaelson MD, Gorbunova VA, Gore ME, Rusakov IG, Negrier S, Ou YC, Castellano D, Lim HY, Uemura H, Tarazi J, Cella D, Chen C, Rosbrook B, Kim S, Motzer RJ. Comparative effectiveness of axitinib versus sorafenib in advanced renal cell carcinoma (AXIS): a randomised phase 3 trial. Lancet. 2011; 378:19311939. DOI: 10.1016/S0140-6736(11)61613-9 [PubMed: 22056247]

15. Rixe O, Bukowski RM, Michaelson MD, Wilding G, Hudes GR, Bolte O, Motzer RJ, Bycott P, Liau KF, Freddo J, Trask PC, Kim S, Rini BI. Axitinib treatment in patients with cytokinerefractory metastatic renal-cell cancer: a phase II study. Lancet Oncol. 2007; 8:975-984. DOI: 10.1016/S1470-2045(07)70285-1 [PubMed: 17959415]

16. Tomita Y, Uemura H, Fujimoto H, Kanayama HO, Shinohara N, Nakazawa H, Imai K, Umeyama Y, Ozono S, Naito S, Akaza H. Key predictive factors of axitinib (AG-013736)-induced proteinuria and efficacy: a phase II study in Japanese patients with cytokine-refractory metastatic renal cell Carcinoma. Eur J Cancer. 2011; 47:2592-2602. DOI: 10.1016/j.ejca.2011.07.014 [PubMed: 21889330]

17. Rini BI, Wilding G, Hudes G, Stadler WM, Kim S, Tarazi J, Rosbrook B, Trask PC, Wood L, Dutcher JP. Phase II study of axitinib in sorafenib-refractory metastatic renal cell carcinoma. J Clin Oncol. 2009; 27:4462-4468. DOI: 10.1200/JCO.2008.21.7034 [PubMed: 19652060]

18. Huang SM, Temple R, Xiao S, Zhang L, Lesko LJ. When to conduct a renal impairment study during drug development: US Food and Drug Administration perspective. Clin Pharmacol Ther. 2009; 86:475-479. DOI: 10.1038/clpt.2009.190 [PubMed: 19844224]

19. Xu XS, Yuan M, Karlsson MO, Dunne A, Nandy P, Vermeulen A. Shrinkage in nonlinear mixedeffects population models: quantification, influencing factors, and impact. AAPS J. 2012; 14:927936. DOI: 10.1208/s12248-012-9407-9 [PubMed: 22993107]

20. Savic RM, Karlsson MO. Importance of shrinkage in empirical bayes estimates for diagnostics: problems and solutions. AAPS J. 2009; 11:558-569. DOI: 10.1208/s12248-009-9133-0 [PubMed: 19649712]

21. Committee for Medicinal Products for HUman Use (CHMP). [Accessed 10 April] Note for guidance on the evaluation of the pharmacokinetics of medicinal products in patients with impaired renal function. European Medicines Agency. 2004. http://www.ema.europa.eu/docs/ en_GB/document_library/Scientific_guideline/2009/09/WC500003123.pdf

22. Khosravan R, Toh M, Garrett M, La Fargue J, Ni G, Marbury TC, Swan SK, Lunde NM, Bello CL. Pharmacokinetics and safety of sunitinib malate in subjects with impaired renal function. J Clin Pharmacol. 2010; 50:472-481. DOI: 10.1177/0091270009347868 [PubMed: 19779038]

23. Smith W, Marbury T, Kipnes M, Cihon F, Lettieri J, Mazzu A. Effects of renal impairment on the pharmacokinetics of sorafenib and its metabolites. Cancer Res. 2007; 67:934.

24. Gupta S, Parsa V, Heilbrun LK, Smith DW, Dickow B, Heath E, Vaishampayan U. Safety and efficacy of molecularly targeted agents in patients with metastatic kidney cancer with renal dysfunction. Anticancer Drugs. 2011; 22:794-800. DOI: 10.1097/CAD.0b013e328346af0d [PubMed: 21799472]

25. Kim KH, Kim HY, Kim HR, Sun JM, Lim HY, Lee HJ, Lee S, Bae WK, Rha SY. Efficacy and toxicity of sunitinib in patients with metastatic renal cell carcinoma with renal insufficiency. Eur J Cancer. 2014; 50:746-752. DOI: 10.1016/j.ejca.2013.11.029 [PubMed: 24332573]

26. Josephs D, Hutson TE, Cowey CL, Pickering LM, Larkin JM, Gore ME, Van Hemelrijck M, McDermott DF, Powles T, Chowdhury P, Karapetis C, Harper PG, Choueiri TK, Chowdhury S. Efficacy and toxicity of sunitinib in patients with metastatic renal cell carcinoma with severe renal impairment or on haemodialysis. BJU Int. 2011; 108:1279-1283. DOI: 10.1111/j.1464-410X. 2010.09990.x [PubMed: 21244613]

27. Shetty AV, Matrana MR, Atkinson BJ, Flaherty AL, Jonasch E, Tannir NM. Outcomes of patients with metastatic renal cell carcinoma and end-stage renal disease receiving dialysis and targeted therapies: a single institution experience. Clin Genitourin Cancer. 2014; 12:348-353. DOI: 10.1016/j.clgc.2014.01.004 [PubMed: 24565697]

28. Masini C, Sabbatini R, Porta C, Procopio G, Di Lorenzo G, Onofri A, Buti S, Iacovelli R, Invernizzi R, Moscetti L, Aste MG, Pagano M, Grosso F, Lucia Manenti A, Ortega C, Cosmai L, 
Del Giovane C, Conte PF. Use of tyrosine kinase inhibitors in patients with metastatic kidney cancer receiving haemodialysis: a retrospective Italian survey. BJU Int. 2012; 110:692-698. DOI: 10.1111/j.1464-410X.2012.10946.x [PubMed: 22364110]

29. SUTENT ${ }^{\circledR}$ (sunitinib malate) prescribing information. Vol. 2014. Pfizer Inc; New York, NY, USA: 2014.

30. VOTRIENT (pazopanib) prescribing information. GlaxoSmithKline; Research Triangle Park, NC: 2014. 


\section{Key points}

- A population pharmacokinetic model showed axitinib pharmacokinetics were similar regardless of baseline renal function, and analyses from clinical studies in patients with metastatic renal cell carcinoma suggested impaired renal function did not impact tolerability of axitinib.

- $\quad$ These data indicate that adjustments of the standard starting dose of axitinib (5 mg twice daily) are not needed in patients with mild to severe renal impairment. 


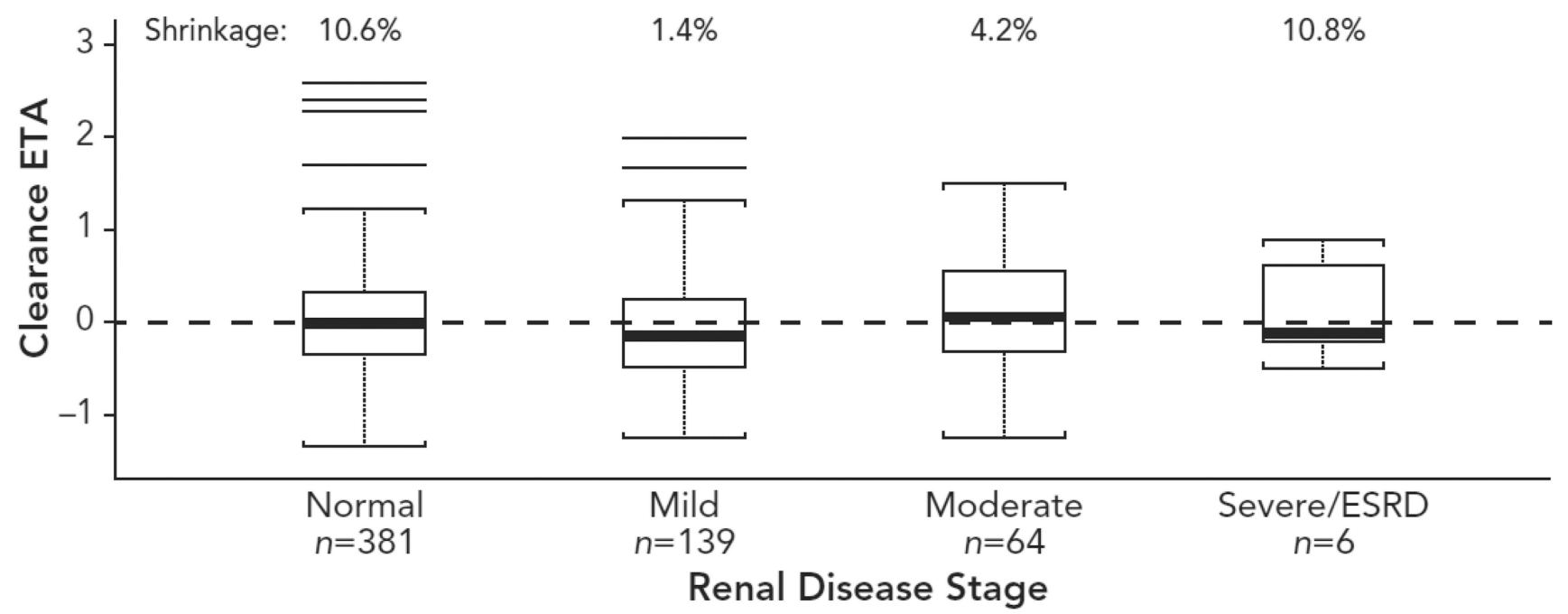

Fig. 1.

Final model estimated axitinib clearance according to renal function (baseline creatinine clearance). Shrinkage refers to the phenomenon that occurs when a model is overparameterized for the amount of information contained in the data. ESRD, end-stage renal disease; ETA, empirical Bayes prediction of the interindividual random effect. 


\section{롤 \\ }

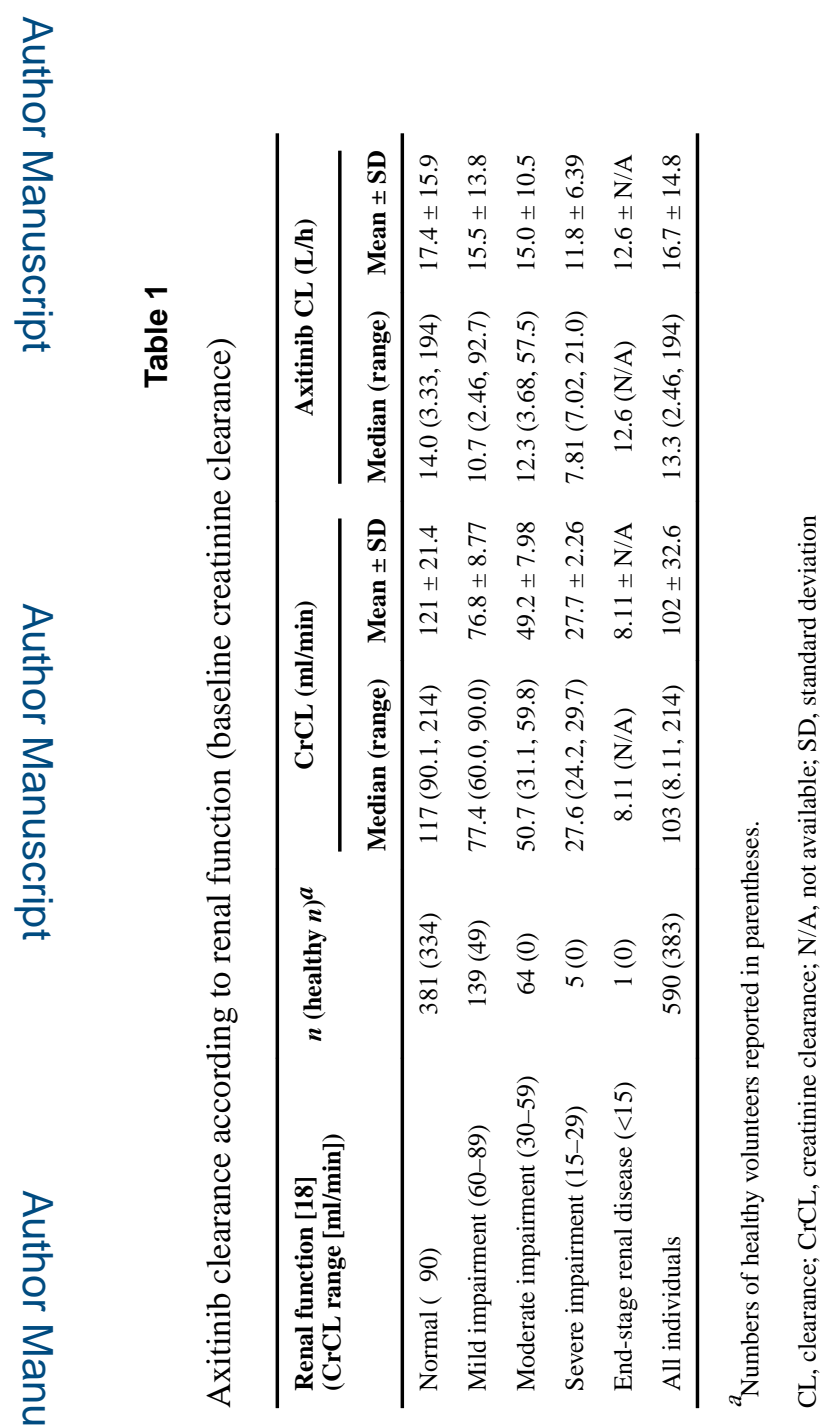

Target Oncol. Author manuscript; available in PMC 2017 August 23. 


\section{Table 2}

Changes in serum creatinine or creatinine clearance from baseline according to renal function (baseline creatinine clearance)

\begin{tabular}{|c|c|c|}
\hline \multirow{2}{*}{$\begin{array}{l}\text { Renal function [19] } \\
\text { (CrCL range [mL/min]) }\end{array}$} & Change in serum creatinine $\mathrm{e}^{a}(\mu \mathrm{mol} / \mathrm{L})$ & Change in $\mathrm{CrCL}^{b}(\mathrm{ml} / \mathrm{min})$ \\
\hline & Median (range) & Median (range) \\
\hline Normal or mild impairment ( 250$) ; n=271$ & $14.0(-53.0,154)$ & $-8.84(-77.4,55.6)$ \\
\hline Moderate impairment (30-50); $n=67$ & $17.0(-26.0,98.1)$ & $-5.59(-19.3,9.38)$ \\
\hline Severe impairment $(<30) ; n=3$ & $19.0(11.5,53.0)$ & $-3.47(-6.94,-3.42)$ \\
\hline
\end{tabular}

${ }^{a}$ Highest serum creatinine at any visit minus baseline serum creatinine.

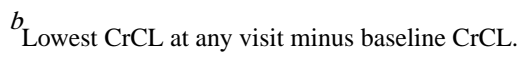

$\mathrm{CrCL}$, creatinine clearance 